\title{
Dedicated Detection Algorithms for Automatic Fire Detection
}

\section{HEINZ O. LUCK}

Duisburg University, Department of Communication Engineering

Bismarckstr. 81, D-4100 Duisburg, Germany

\begin{abstract}
:
Automatic fire detection systems can be made very sensitive to detect genuine fires. But in the practical application they often suffer from unacceptable high false alarm rates that are due to detector deception in about $50 \%$ of the cases. To improve the situation it is necessary to use as much information contained. in the sensor signal of the detector head as possible. Modern software controlled electronics offer the opportunity to implement even more sophisticated signal processing algorithms and to apply them in practical installations. So the development of effective detection algorithms that are dedicated to fire detection problems becomes increasingly interesting. The contribution deals with fundamental features that can be observed in sensor signals from fire detector heads in practical installations and with the development of detection algorithms based on these observations. Serveral different proposals recently made in the literature are discussed. Single sensor based fire detection (SSbFD) as well as multiple sensor based fire detection (MSbFD) is mentioned. In the MSbFD case spot type MSbFD-systems and space type MSbFD-systems are considered.

The chances for an application of fire detection algorithms of the dicussed type in commercial applications depend very much on the possibility of testing the new systems with an accepable expenditure of time and money.
\end{abstract}

REYWORDS: Fire detection; Detection algorithms; Fire signal analysis.

\section{INTRODUCTION.}

Detection is the condition for any countermeasure against a developing fire. Automatic fire detection is based on a careful monitoring of so-called fire parameters such as temperatur, "smoke density" measured by different methods (optical scattering or ionisation chamber method in most of the practical cases), flame radiation in different wavelength bands etc. Early detection of a real developing fire is not an unsolved technical problem. The problem involved is detectivity, i.e. the ability of an automatic system reliably to distinguish between the FIRE and the NOT FIRE case.

Automatic fire detection can be considered as a special type of signal detection where the signal to be detected is not known in advance. Starting fire show a wide range of different forms of appearance, e.g. smouldering, flaming, slow or rapid development. On the other hand combustion of different kinds like 
smoking, heating, cooking, car- and truck-driving or combustion in nearly every labrication process is present almost everywhere in our environment. For these reasons changes in all technically usable fire parameters have mainly not uniquely recognizable sources. In addition electronic noise, weather and other environmental conditions (heat, air pressure, humidity etc.) influence the measured $m(t)$, see fig. 1, directly or indirectly, e.g. by an impact on the sensing element.

These reasons alltogether produce roughly speaking one half of the false alarm problem in practice [1]. The other half is due to inappropriate installation design, electrical and other failures in the installation, unqualified operation and maintenance [2]. The false alarm problem is important for practical applications because the false alarm rate is mainly above 10:1 (false alarms to genuine fire alarms), and this fact gives rise to considerable doubts against the technique of automatic fire detection at all. The main intention to consider better and more reliable detection procedures in this context is to reduce the false alarm rate. It has to be emphasized, that the detection algorithm applied can only control the one half of the problem mentioned above.

The following contribution deals with this first part only in that it considers the fire detection as a special signal detection problem. The task is to extract as much information as possible about the sources of variation of the fire parameter $x(t)$ from the available measured values $m(t)$.

\section{FIRE DETECTION AS A SPECIAL SIGNAL DETECTION} PROBLEM.

Fig. 1 shows a schematic sketch of the detection procedure. $x(t)$ is some physical parameter that undergoes a variation if a fire is developing in the vicinity of the detector head. But no variation of any $x(t)$ is known that is undoubtly and uniquely due to a first stage of a developing fire. Other sources may produce similar variations as well. Nor is the particular shape of the time function $\mathrm{x}(\mathrm{t})$ or its magnitude uniquely determined by a fire situation in the premises that is to be protected. The location of an ignition is by no means to be predetermined with respect to the detector head location. Several existing dispersion models for heat and smoke may be applied but they cannot really help in this situation mainly because detection has to take place in the very first extremly nonstationary phase of the fire development. In addition other parameters in the environment including other heat and combustion sources may control the "fire parameter" $x(t)$ to a considerable extent.

So fire detection in comparison with other classical signal detection problems shows the difficulty that the signals to be detected

- are not known or determinable in advance and

- may be different to an considerable amount if slowly smouldering fires as well as rapidly growing flame fires are taken into account.

In a small minority of practical cases it is known what kind of fire development is likely to occur.

Although the signals to be detected are considerably different they may have common features in some characteristic details of the time functions $x(t)$ or their frequency spectra. It is the task for the signal processing $T[m(t)]$ to extract these features and form a processed value $y(t)$ so that a decision making device, i.e. a simple threshold comparison $y(t) \geq S$, results in a distinct decision $D(y)=1$ or 0 for FIRE or NOT FIRE respectively.

As can be seen from fig. 1 the fire parameter $x(t)$ is not available for further processing. Each sensor converts $x(t)$ into a measured value $m(t)$ which is the one available for processing. $x(t)$ and $m(t)$ are of course not identical. 


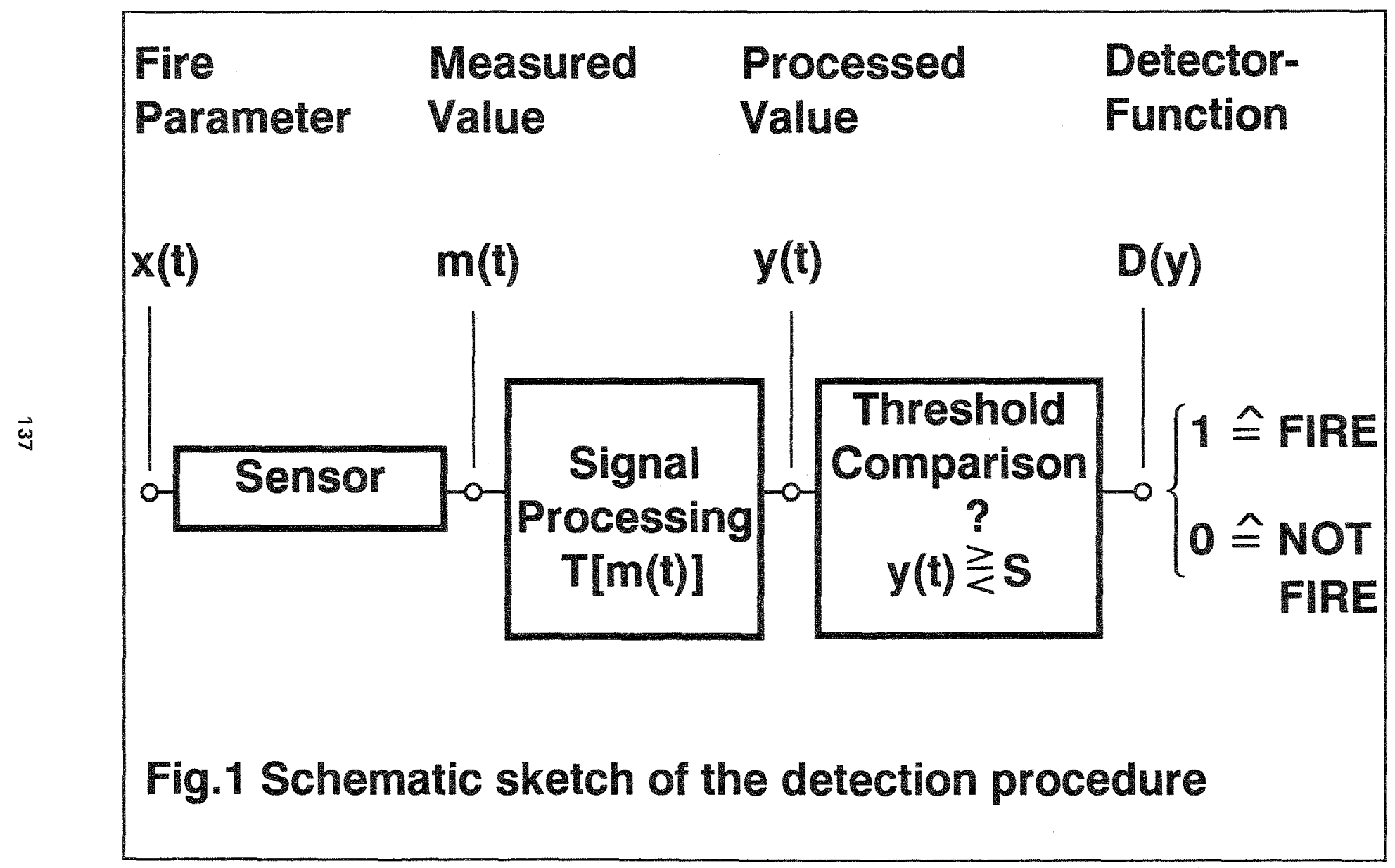


If the sensor is a meaningful device $m(t)$ is predominantly controlled by $x(t)$. But there are several other effects which may be characterized as follows:

- Physical characteristics of the sensor element. Mainly it has a low pass filtercharacteristic due to its physical principle and its mechanical design. Smoke sensors have a special characteristic due to the smoke penetration behavior into the measuring chamber. In the flame detector case screening effects and/or contributions from wall reflected signals as well as frequency and wavelength characteristics are of importance.

- Environmental impacts on the sensor. Temperatur variations, humidity, air pressure often have a measurable influence on the sensor performance.

- Drift effects on the sensor output signal, which are due to pollution or contamination of the seasing element.

- Electronic noise.

All these effects result in a measurement noise that superimposes the effect of $x(t)$ on $m(t)$. Having this in mind the most simple model for $m(t)$ is :

$m(t)=m_{x}(t)+m_{n}(t)$

with

and

$$
m_{X}(t)= \begin{cases}0 & \text { in the NOT FIRE case } \\ m_{X}(t) & \text { in the FIRE case }\end{cases}
$$

$\mathrm{m}_{\mathfrak{n}}(\mathrm{t})$ not effected by a fire in the vicinity of the detector head. But it may produce signal variations in $m(t)$ of the same magnitude as $\mathrm{m}_{\mathrm{X}}(\mathrm{t})$.

In the FIRE case it is not possible to measure both components separately. In the NOT FIRE case $m_{\mathfrak{n}}(t)$ can be measured as a random process. For further processing $m(t)$ itself has therefore to be treated as a random signal. Classical or new signal processing tools can be used to extract the necessary information. Therefore it is necessary for fire researchers, who deal with detection problems, to recognize signal processing means, i.e. to understand them at least as good as the involved measurement tools. Otherwise good possibilities for early detection will be ignored, that may become applicable for the following reasons:

- The modern development in the mathematics of signal processing algorithms (correlation methods, signal prediction theory, optimal filtering etc.) offers new applications in the fire detection technique too.

- Modern electronic hardware and software facilities create the opportunity to implement fast and effective detection algorithms without the need for increasing the system prices in an unsuitable way.

On the other hand basic knowledge about fire protection is necessary in the development process for new fire detection tools and therefore it is not suitable to get this work done by electronic engineers or data processing experts alone.

\section{FUNDAMENTAL FEATURES FOR PIRE DETECTION \\ ALGORITHMS.}

Fire detection algorithms have to be based on the measured $m(t)$. No meaningful general model that gives more details than formula (2.1) has been dicussed so far. That means that every detailed model or algorithm is dedicated to the measurement procedure which is used to produce that particular $m(t)$ value. In this sense all the algorithms $T[m(t)]$ which are used for further processing are "dedicated algorithms". 
The algorithm designed to process a temperature measurement is completely different from one to process a smoke-density measurement or a signal from a IR- or UV-radiation measurement.

It is therefore necessary very carefully to study the signals for different cases of fire parameter measurements. This has to be done in both the FIRE and the NOT FIRE case. The first is comparably easy because a series of test fires in a fire research laboratory is not so difficult to control. The latter is far more difficult because an enumerous variety of different situations has to be taken into account. In any case the search for fire detection algorithms $T[\mathrm{~m}(\mathrm{t})]$ has to be based on the knowledge of the essential features of measured values in the field. In several places such measurements in the field have been done or are under work and some material is already available [3].

Fig. 2 shows some examples of measured values from three different fire parameters. The signals are plotted in an arbitrary but equal scale over a time period of six minutes. In Fig. 2a a typical FIRE situation is documented whereas Fig. $2 b$ shows a NOT FIRE event in a canteen kitchen of a hospital. It is obvious that in both situations the same magnitude of variation for measured values are produced. In the NOT FIRE case a false alarm would have been produced if e.g. the optical smoke sensor signal alone would have been monitored. Although the situation shown in Fig. $2 b$ is a rare event it can be seen that the magnitude of the measured value $m(t)$ cannot be the distinguishing feature at least not the distinguishing feature alone. On the other hand the corresponding signal plots are by no means really similar. Therefore it should be possible to find and to formulate signal features or combinations of signal features that are reliably different in both the FIRE and the NOT FIRE case. These differences will remain even if different FIRE cases and a wide variety of NOT FIRE cases are taken into account. This means that a careful signal analysis is necessary.

In this context it should be mentioned that we deal with a pure detection problem. It is not the intention to recover the signal shape but to detect the fact whether or not $m(t)$ contains some kind of $m_{x}(t)$ or not.

A first rough check of the available material yields the following result that partly also can be derived from the plots in Fig. 2 :

- All signal plots can be considered as a combination of a slowly varying (low pass) component and a rapidly varying component both of irregular shape. That means that all the signal plots of measured values $m(t)$ are sample functions of nonstationary random signals.

- Signals measured in the NOT FIRE situation show long time periods with very small variations. They are randomly followed by periods with definitely nonstationary time behavior like that shown in Fig. $2 \mathrm{~b}$.

- Signals from different sensors seem to be correlated in a different way in the FIRE and in the NOT FIRE case.

- Signal trends of different kinds can be observed.

- The fluctuations of signals in the FIRE case are not as characteristic as it is is to be expected in rapidly growing fire situations.

\section{SINGLE SENSOR BASED PIRE DETECTION (SSbFD).}

The mainly used detection method in practice is based on the measured signal $m(t)$ from one single sensor. A rough examination of measured signals $m(t)$ shows some signal features that are more or less characteristic for the FIREor for the NOT FIRE case. Although they are not definitely unique for every possible situation they may serve as distinguishing features. Before studying this in detail we have to keep in mind the following facts :

- The time period for detection of a real fire is limited to at most a few minutes. 

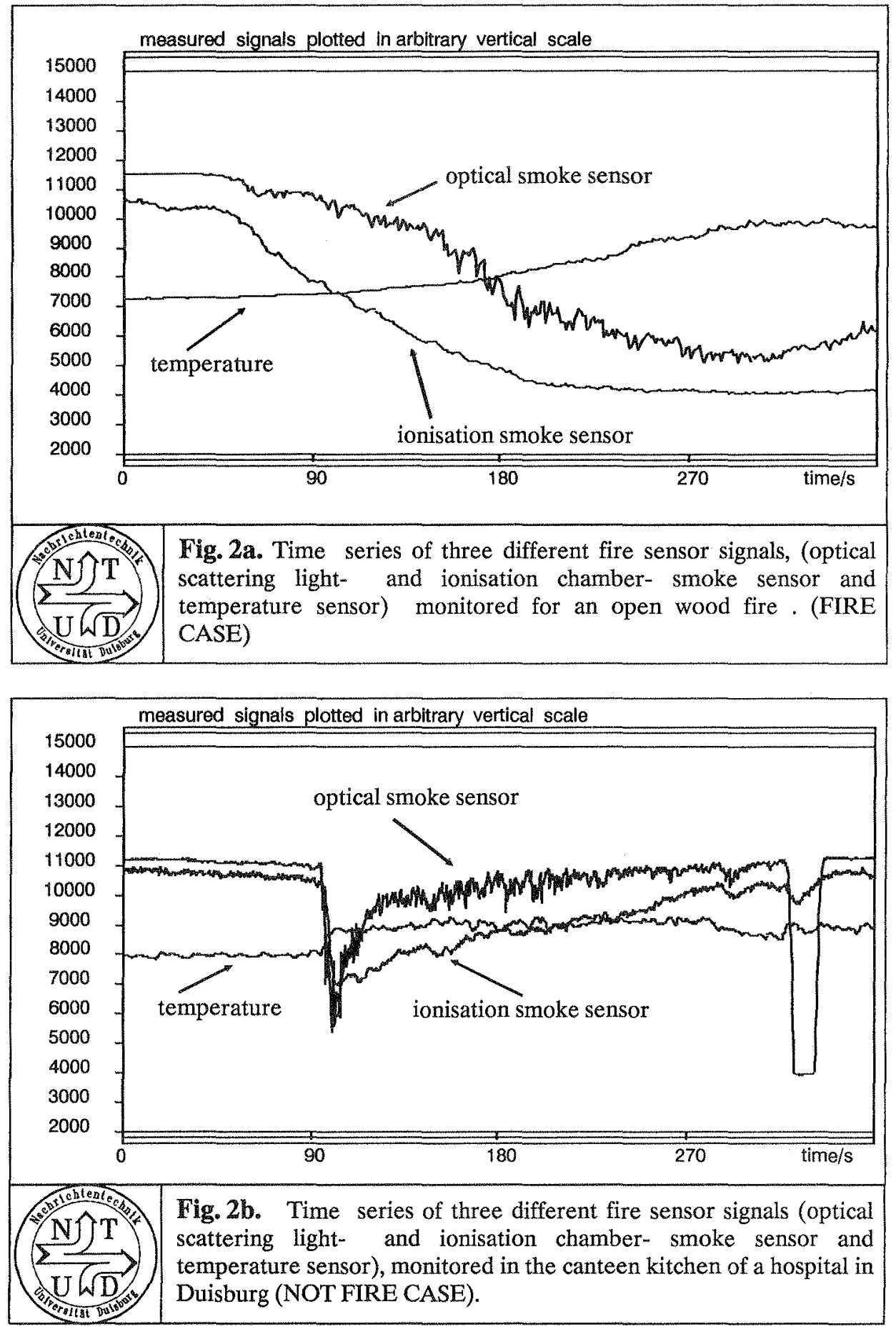
Let us call this time period $\theta$. It is different from the memory time $t_{n}$ that a detection algorithm $T[m(t)]$ may have.

- The signals that are to be observed are random signals in both the FIRE and the NOT FIRE case.

Some signal features to be extracted from the measured signal values $m(t)$ and the associated detection algorithms are dicussed in the following.

4.1 The majority of the measured signals $\mathrm{m}(\mathrm{t})$ show a more or less significant trend that is positive (increasing signal) or negative (decreasing signal) in the FIRE case during the whole fire burning period and therefore also in all suitable detection periods $\Theta$. A positive trend e.g. means that the majority of values $m\left(t_{0}+t\right)$ following any instant value $m\left(t_{0}\right)$ is greater than this instant value and vice versa.

There are several different trend detection algorithms known from the literature [4]. The most suitable one for practical implementation is the Kendall- $\tau$-detector [5] mainly because it can be written in a recursive form.

$y(k)=\sum_{i=0}^{n-1} \sum_{j=i}^{n-1} u[m(k-j)-m(k-i)] \lesseqgtr s$

where $S$ is the detection threshold and $k$ is a discrete time variable that is used instead of $\mathrm{k} \cdot \Delta \mathrm{t}$ in order to shorten the notation. $\Delta \mathrm{t}$ is a suitable sampling period. The recursive form reads

$y(k)=y(k-1)-\sum_{1=1}^{n-1} u[m(k-(n-1))-m(k-n)]+\sum_{i=1}^{n-1} u[m(k)-m(k-(n-i))]$

and

$u(\xi)=\left\{\begin{array}{l}1 \text { for } \xi>0 \\ 0 \text { for } \xi \leq 0\end{array}\right.$.

The main properties of this kind of signal detector are dicussed in the literature [5,6]. It should be mentioned that it is fast and simple in that a comparison procedure is only followed by an addition of zeros and ones. The Kendall- - -detector and all other known trend detection algorithms do not rate the magnitude of the signal's increase or decrease resp. They just give an indication for the direction of trend, i.e. positive or negative. Formula (4.1) shows the algorithm for positive trend detection. Negative trends can be measured using the same algorithm by only multiplying $\mathrm{m}(. .$.$) by -1$. The Kendall- $\tau$-detector and of course other trend detectors too depend very much on the actual window length $n$ or $n \cdot \Delta t$ used for the observation of data. Too small (in the order of seconds) and too large (in the order of an hour or more) window lengths may cause too many false alarms. The first give a reaction due to noisy signal fluctuations, the latter measure the trend due to climatic changes or night and day variations.

Trend detection as a single sensor based detection procedure alone cannot serve as a reliable fire detection tool. From the signal records in NOT FIRE cases it can be seen that fairly often the measured signal values $m(t)$ show smooth long term trends due to climate or other environmental influences. But it is very usefull as a component in a multiple-algorithm detection procedure [6] or as a detection algorithm in a MSbFD. 
4.2 In nearly all FIRE situations the slowly varying (low pass) component of the measured signal $\mathrm{m}(\mathrm{t})$ tends to exceed a predetermined threshold for a considerable longer time period as in NOT FIRE situations (see fig. 2). This introduces the idea that not the signal magnitude is essential for the FIRE case but it's integral during the period when $m(t)$ exceeds a threshold $\mathrm{M}_{\mathrm{S}}$. The following general detection algorithm results :

$y(k)=K_{0} \sum_{i=0}^{n-1} w\left(k_{g} i\right) \cdot\left[m(k-i)-M_{s}\right] \cdot u[m(k-i)-M s] \lessgtr s$

where $w(k, i)$ is a suitable weight function.

$\mathrm{K}_{0}$ is a constant that may be used to normalize the $\mathrm{y}(\mathrm{k})$ value.

$\mathrm{M}_{S}$ is a predetermined threshold for the measured signal $\mathrm{m}(\mathrm{t})$.

$\mathrm{S} \quad$ is again the detection threshold.

(4.4) is a biased filter algorithm the character of which is determined by the weight factors $w(k, i)$. The main advantage of this algorithm is that a single value or few single values $m(k)$ that may be erroneous and of high magnitude do not influence the final result in a decisive way if the observation window length $\mathrm{n}$ is choosen appropriately. The algorithm is computationally simple and therefore easy to implement.

An example for a fire detection algorithm of the (4.4)-type is the so-called "Brandmengen"-detector that is already used in commercially available fire detection systems [7]. In this case the weight factors are simply equal and constant and the observation window length is made flexible i.e. dependent on the measured values $\mathrm{m}(\mathrm{k})$.

$\mathrm{y}_{\mathrm{B}}(\mathrm{k})=\left[\mathrm{y}_{\mathrm{B}}(\mathrm{k}-1)+\mathrm{K}_{0}\left(\mathrm{~m}(\mathrm{k})-\mathrm{M}_{\mathrm{S}}\right)\right] \cdot \mathrm{u}\left[\mathrm{m}(\mathrm{k})-\mathrm{M}_{\mathrm{S}}\right] \lessgtr \mathrm{S}$

So the output $y_{B}(k)$ is accumulated as long as $m(k)$ exceeds the threshold $\mathrm{M}_{\mathrm{S}}$. Otherwise it is set to zero. In addition a known practical application provides a threshold value $M_{S}$ that is adaptive to the environmental conditions to some extend [8]. Detectors of this kind can be characterized as biased integrating detectors.

4.3 A careful examination of recorded data in the FIRE and in the NOT FIRE case shows that the character of the observed data is changed in a random but recognizable way if the situation changes from one case to the other. It seems to be obvious that some characteristic combinations of features like trends, kind of fluctuation, fluctuation speed etc. do exist in a predetermined way. On the other hand the measured signals $\mathrm{m}(\mathrm{t})$ obviously keep their random behaviour with only piecewise stationary periods. In this case detection procedures based on the autocorrelation-function or the power-spectral-density may be suitable. In other words such a procedure is based on the second order statistics of the observed signal.

Klose and Siebel recently proposed a fire detector based on the comparison of the short-term autocorrelation-function $R_{\mathrm{mm}}(\tau)$ of the observed data $\mathrm{m}(\mathrm{t})$ with the corresponding correlation-function $\mathrm{R}_{\mathrm{m}} \mathrm{R}^{\mathrm{R}}(\tau)$ of a reference signal $\mathrm{m}^{\mathrm{R}}(\mathrm{t})$ or their power-spectral-density functions $S_{\mathrm{mm}}(\omega)$ and $\mathrm{S}_{\mathrm{m}} \mathrm{R}_{\mathrm{m}} \mathrm{R}(\omega)$ respectively [9]. Both signals are considered to be wide sense stationary within the observation period $q \cdot \Delta t$ and frequency-band limited to $\omega_{\mathrm{g}}$, where $\Delta t$ is a suitable sampling period following the Nyquist condition. The test statistic is simply the square sum of the difference between the power-spectral- density functions over the frequency band $\omega_{\mathrm{g}}$. 
$\mathrm{y}=\int_{-\omega \mathrm{g}}^{+\omega}\left(S_{\mathrm{mm}}(\omega)-S_{\mathrm{m}^{\mathrm{R}} \mathrm{m}} \mathrm{R}(\omega)\right)^{2} \mathrm{~d} \omega \lesseqgtr \mathrm{S}$

This algorithm can be rewritten in a form that can be calculated from the input data (see [9])

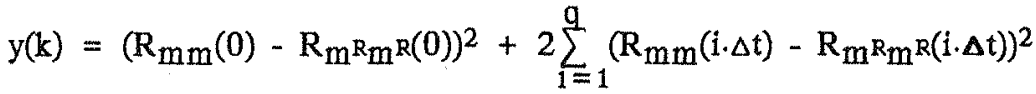

where the $R_{m m}(\ldots)$ and $R_{m} R_{m} R(.$.$) values are calculated directly from the$ input data as an correlation function estimate. The only problem to be solved is to find a suitable reference signal $\mathrm{m}^{\mathrm{R}}(\mathrm{t})$. This is done in the following way. The second order statistics of any measured $m(t)$ are different in the FIRE and in the NOT FIRE case. So they will undergo a more or less rapid change if the situation changes from NOT FIRE to FIRE. The longer the window which is used to calculate the correlation function estimates from the input data the more slowly this change will influence the calculation result, in other words : A short window is used to calculate $R_{m m}(\tau)$, that means a quick reaction on a changing situation, and a much larger window is used to calculate $R_{\mathrm{m}} \mathrm{Rm} R(\tau)$ from the same input data. This means that the input data itself is used as a reference signal by taking much more of its past values into account. In fact the comparison is made between the second order statistics of the same input signal but different parts on the time scale of this signal are considered [9]. The computational effort of a detection algorithm like this is comparably high. It has been tested already and the results obtained were very promissing. As this detection method is applicable in a multiple sensor based fire detection (MSbFD) procedure as well it will be shortly discussed later on again.

4.4 A most rigorious way to design a detection algorithm on the background of a high randomness of the measured data $m(t)$ is to develop signal models for the different cases FIRE and NOT FIRE which provide signal samples with the same statistical parameters as the original measured data. These models have to describe the involved signals by a limited number of parameters. These parameters or at least one of them may then be calculated from the actual signal $\mathrm{m}(\mathrm{t})$ measured by the sensor and used for distinction between the two possible situations FIRE and NOT FIRE.

A most powerful method to describe random signals is to model them by a so-called autoregressive(AR)-signal model based on the one step prediction error filter technique [10]. The basic idea of this technique is explained in fig. 3. Fig. 3a shows a one (time)step prediction error filter which is designed to minimize the prediction error signal energy

$E_{e}(t)=\int_{t-n \Delta t}^{t} e^{2}(t) d t \quad$ or $\quad E_{e}(k \cdot \Delta t)=\sum_{i=0}^{n-1} e^{2}((k-i) \cdot \Delta t)$

The prediction filter order $\mathrm{n}$ is choosen to completely decorrelate the filter output $e(t)$ to a white noise process, i.e. the samples $e(k \cdot \Delta t)$ become uncorrelated random variables (for further details see [10] or [11]). Due to Wold's decomposition theorem [10] this result is reversible in the sense that the coefficients $\left\{c_{\nu}\right\}, \nu=[1, \ldots, n]$ can be used to design a so-called 

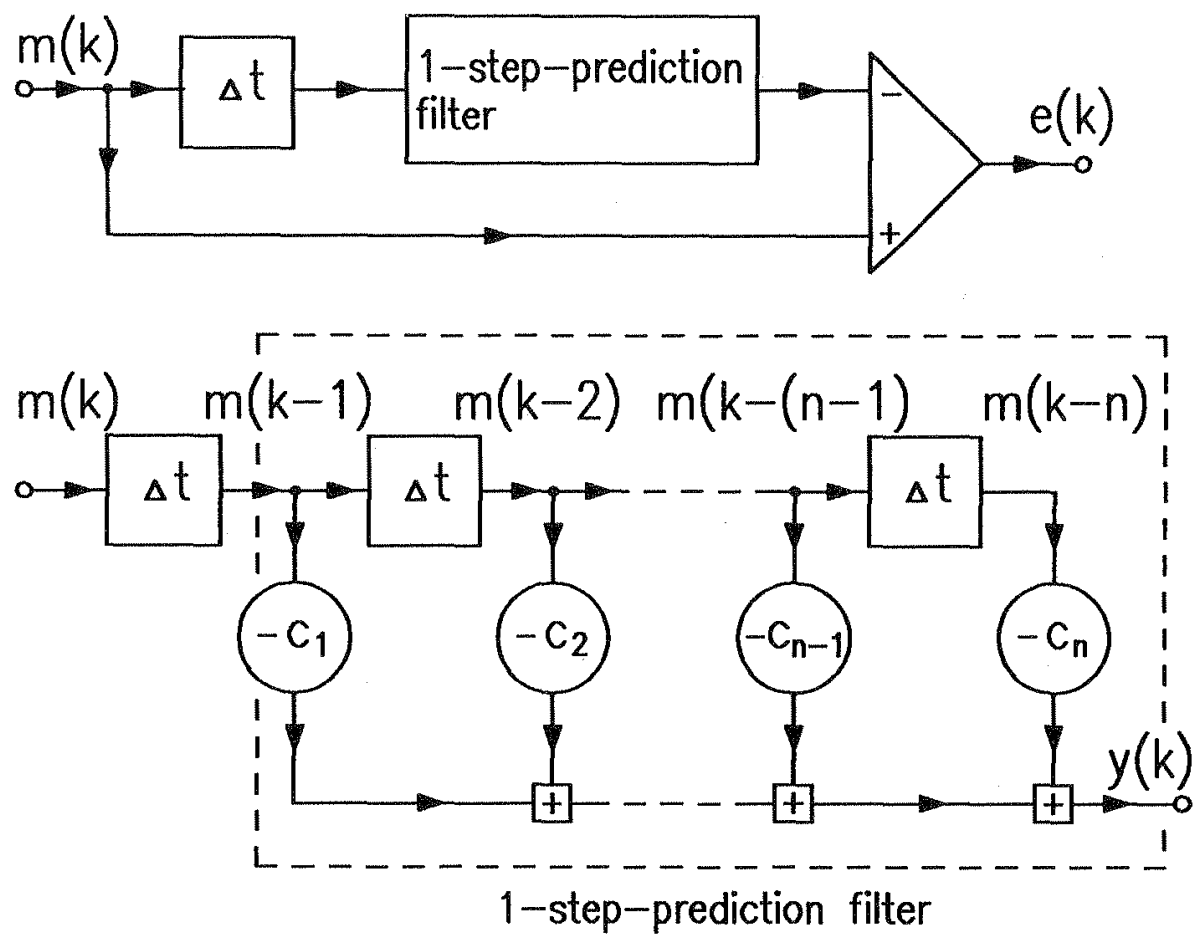

Fig.3a One-step-prediction error filter

autoregressive(AR) signal model as shown in fig. $3 \mathrm{~b}$. In this way the signal $\mathrm{m}(\mathrm{t})$ is completely characterized by the $n+1$ parameters $\left\{c_{\nu}\right\}$ and the prediction error signal energy $E_{e}$ as far as the second order statistics of the signal process are concerned. At least one computationally effective time recursive algorithm is known in the meantime to compute these parameters from the actual input data $m(k)$ on line with good accuracy [12]. Several experiments have shown considerable differences in these parameters for the FIRE and the NOT FIRE case. Consequently a first proposal for a detector algorithm recently has been made by Sokat [12] that is based on the most easy to calculate parameter combination $E_{m}(k \cdot \Delta t)-E_{e}(k \cdot \Delta t)$. 


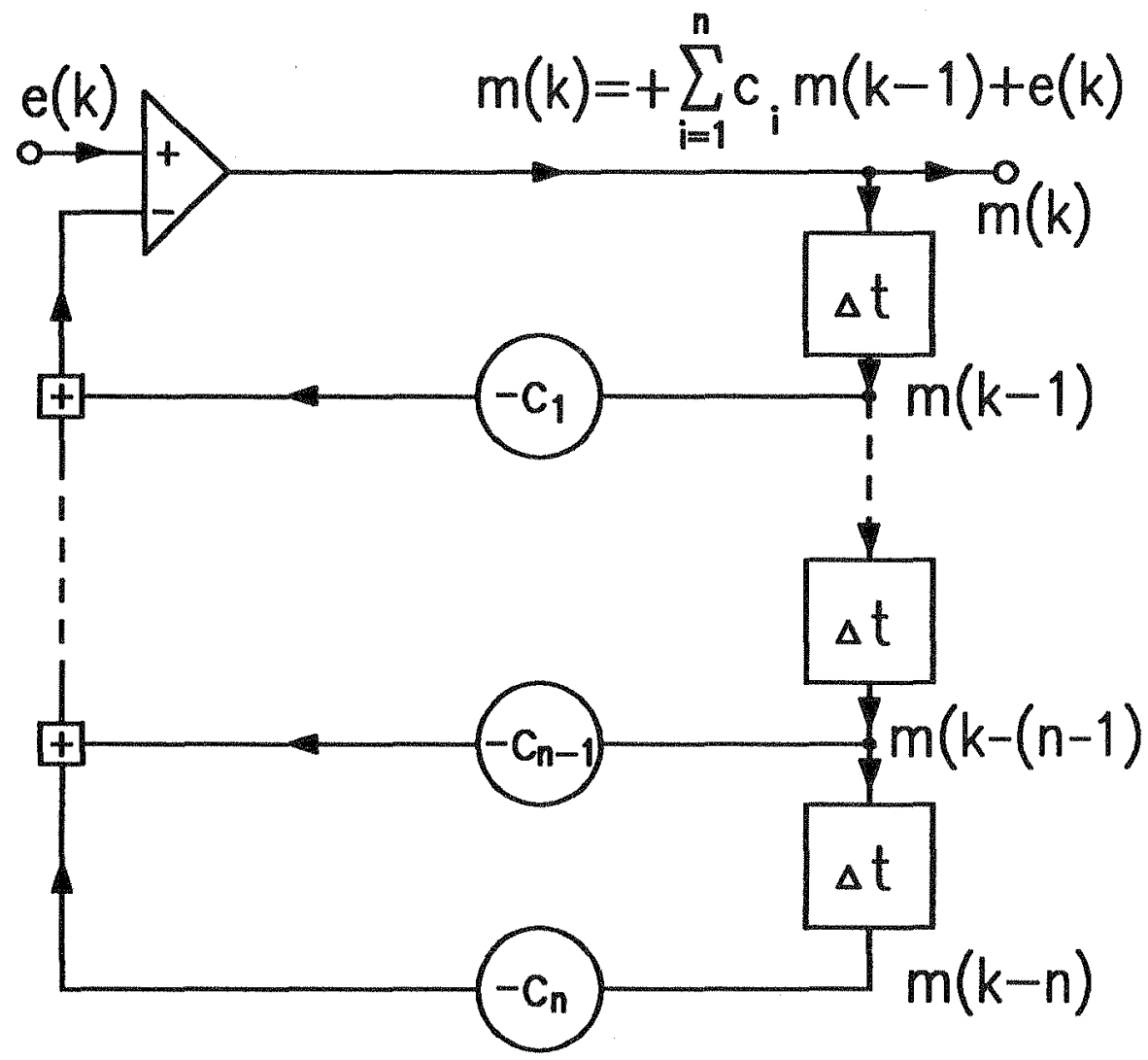

Fig.3b Autoregressive(AR)-signal model

$y(k)=\sqrt{E_{m}} \overline{(k \cdot \Delta t)-E_{e}(k \cdot \Delta t)}$

where

$E_{m}(k \cdot \Delta t)=\sum_{i=0}^{n-1} m^{2}((k-i) \cdot \Delta t)$

is the energy of the actually measured $m(t)$ within the observation time period and $\mathrm{E}_{\mathrm{e}}(\mathbf{k} \cdot \Delta \mathrm{t})$ is the associated prediction error signal energy.

The computational effort for this detection algorithm is comparably high. But it is easily applicable in MSbFD systems as well and will be discussed under this item again.

This list of possible types of fire detection algorithms is by no means complete. Only those were discussed that are apparent in the actual literature. 


\section{MULTIPLE SENSOR BASED FIRE DETECTION (MSBPD).}

A very attractive idea to improve the detectivity of automatic fire detection systems is to use more than one different or differently located sensors to form the processed value $y(t)$ (see fig. 1). Consequently we have to distinguish between:

- Spot type multiple sensor based fire detection and

- Space type multiple sensor based fire detection.

In general any logic network combination of SSbFD-algorithms can be used to design a MSbFD-system. In this case the detection procedures including the threshold comparison part are left unchanged (see fig. 1) and only the final decision results are combined by e.g. a logic "And"-procedure. Several examples of this technique are already known from practical applications.

One example often used in order to control the false alarm behavior is the socalled "two line dependence"-technique. Here a fire alarm is only released if two different lines in a fire detection installation independently indicate an alarm situation. This method is mainly applied in systems that automatically release an extinguishing agent and therefore may cause considerable damage in a false alarm situation. This application is mainly a space-type-MSbFD.

Other examples are flame detectors which combine wave length selective IRcomponents and UV-components by a logic "And"-network or other means to detect special features of fast growing flames. These are examples for spot-typeMSbFD. Other spot-type-MSbFD systems have been proposed in the literature that use the outputs of different detection algorithms applying a logic network. Here the different algorithms work on the same measured value $m(t)$ [6].

Some recent proposals are worthwhile to shortly be discussed in some more detail:

5.1 Siebel proposed a modified two-input, i.e. two sensor based, Kendall- $\tau$-detector which takes a strong correlation of signal trends in the FIRE case into account that is not given under normal NOT FIRE conditions. The details can be taken from [13]. Here experimental and testing results achieved under FIRE and under NOT FIRE conditions are reported and the behavior of this detector as a smoke detector as well as its limits are discussed.

5.2 Klose and Siebel propose in [9] a MSbFD-system which is based on the detection algorithm already discussed under item 4.3. The multiple sensor structure is simply achieved by summing up several different measured signal values $m_{j}(t)$ or $m_{j}(k)$ resp.

$z(k)=\sum_{j=1}^{p} w_{j} \cdot m_{j}(k)$

and then after an apprropiate adjustment of the weight factors $w_{j}$ applying (4.7). The proper cholce of the weight factors $w_{j}$ introduces the opportunity to adapt the fire detection system to particular conditions of the fire risk on the one hand and to the environment on the other. The reported experimental results show the capability of this detector when it is applied as a MSbFD-system with two different smoke sensors and one temperatur sensor. The high flexibility is one of the main advantages offered by detection systems like this.

5.3 Ishii, Yamauchi et.a1. [14] investigated and proposed a MSbFD-system that is based on the following consideration. The method uses a combined measurement of temperature, smoke density and gas(CO)-concentration. A zone model approach is applied to relate the kind and the size of a fire in 
the compartment to be protected with the measurements that are to be expected in this compartment. With this model an algorithm is established that is used for a reversed application. From the actual measurements of temperature, smoke density (optical extinction coefficient) and COconcentration an estimate is calculated for a heat release rate $Q_{f}$, a smoke generation rate $Q_{s m k}$ and a CO generation rate $Q_{c o}$ that may have caused the measured values. Between any two of these generation rates Q . different correlations do exist depending on the kind of source that is responsible for the actual measurement readings. The associated crosscorrelation coefficients are calculated using a weight factor to adjust the three parameters according to their importance and used as the processed value $y$ (see fig. 1) for detection. In the reported experimental and testing procedure the heat release rate $Q_{f}$ versus the species release rate $Q_{s m k}$ correlation coefficient is examined for several FIRE and NOT FIRE conditions. They show a strong correlation for flaming fire sources and little correlation in a controlled fire case (cooking) (see [14]).

The proposal is an interesting MSbFD which relates the measured values $\mathrm{m}(\mathrm{t})$ from ceiling mounted sensors to an estimated fire source. It requires a considerable computation effort which obviously can be handled by a small PC-size computer. It is not quite clear how senitive the method is to an incorrect choice of several parameter values that are needed for the model calculation. The method itself is the only one known that is directly linked to a fire model in the compartment that is to be protected.

5.4 Okayama studies in [15] a comparably simple neural net configuration for MSbFD. The neural net consists of three layers, input-, output- and one hidden layer with at most five neurons and three or two input signals. The neural net configuration needs a training procedure to adjust the weight factors of the connecting strings between the layers. The results of four case studies of this training procedure are presented. In the first study input signals from a temperature sensor, a smoke sensor and a gas sensor are considered while the output also was formed of three neurons representing probability of a fire, fire risk and smouldering fire probability. A second case study relates a two value input (two neurons in the input layer), a photoelektric smoke sensor signal and its rate of rise value, to a two neuron output layer representing the fire probability. A third and a fourth case study consider a sensor signal and the duration of its crossing a predetermined threshold with and without an additional ventilation as input signals versus a fire probability measure as the neural net output. The reported results of this computer study show the possiblity to design and to configurate an applicable neural network for fire detection purposes.

Although not all of the terms used in the study, e.g. "fire risk", are quite clear from the presentation [15] the approach is interesting and the studies indicate that a further investigation may worthwhile to be undertaken.

\section{CONCLUSION AND SUMMARY.}

New fire detection concepts and algorithms are important for the technical development only if they become attractive for the international market. Practical application and the acceptance in the market depend very much on the following conditions.

- They have to be more effective than already existing ones, i.e. at least lower false alarm rates have to be achieved. This has to be proved by a reasonable testing procedure the results of which are available for acceptable costs and 
in a reasonable time. The only way to solve this problem is to develop a suitable simulation technique. Work is under way in field $[3,7]$.

- They have to be easy to operate and easy to maintain.

- High flexibility for adapting different environmental conditions is of considerable importance.

- Suitable prices are essential.

The above mentioned detection algorithms have a real chance to be applied in new developing fire safety systems because modern software controlled electronics offer the oportunity to implement even more sophisticated detection procedures.

\section{REFERENCES.}

[1] Unoki, J.; Watanabe, A.; Sasaki, H.; Overview of Fire Detection in Japan, Proc. 1. Intern. Symp. on Fire Safety Science, Oct. 1985, Gaithersburg MD. USA; C.E. Grant a. P.J. Pagni ed., Springer Verl. Berlin 1985

[2] Luck, H.; Brockmann, Th.; Schlossarek, U.; New Technologies in Automatic Detection and Suppression, in "New Technologies to Reduce Fire Losses"; Grayson, Smith ed. Elsevier 1986

[3] Thilo, P.; Post, O.; Luck, H.; Siebel, R.; Klose, J.; Schlußbericht zum Forschungsprojekt "Aufnahme u. Analyse unterschiedlicher Brandkenngrößenverläufe...." BMFT / RGB 8509/85100, September 1989

[4] Gibson, J.D.; Melsa J.L.; Nonparametric Detection with Applications, Academic Press, New York 1975

[5] Kendall, M.; Rank Correlation Methods, 4th ed; Ch. Griffin \& Co, London 1975

[6] Luck, H.; Correlation Filters for Automatic Fire Detection Systems, Proc. 1. Intern. Symp. on Fire Safety Sience, p. 749-758, see also [1].

[7] Tink1, W.; Brandkenngrößen analog gemessen und analog übertragen, Proc. 9. Intern. Conf. on Automatic Fire Detection ADBE'89; Duisburg University 1989 ; p. $319-338$

[8] v. Tomkewitsch, R.; Fire Detector Systems with 'Distributed Intelligence'. The Puls Polling System, Fire Safety Journal, 6(1983), p.225-231

[9] Klose, J; Siebel, R; A Fire Detection Algorithm Using Second Order Statistics, Proc. 3. Intern. Symp. on Fire Safety Science, Edinburg 1991

[10] Haykin, S; Adaptive Filter Theory, Prentice-Hall,Englewood Cliffs 1986

11) Klose, J.; Analysis, Synthesis and Simulation of Fire Signals as a Tool for the Test of Automatic Fire Detection Systems, Fire Safety Journal 14 (1991); to be published *

[12] Sokat, J; Ein Beitrag zur adaptiven Filtertheorie mit Anwendungen in der automatischen Brandentdeckung, Dr.-Ing. Thesis, Duisburg Univ. Dept. Electrical and Electronic Engineering, 1991

[13] Siebel, R.; A Composite Detection Algorithm Using Signal Trend Information of Two Different Sensors, Fire Safety Journal 14 (1991); to be published

[14] Ishii, H.; Ono, T.; Yamauchi, Y.; Ohtani, S.; An Algorithm for Improving Reliability of Detection with Processing of Multiple Sensor's Signal, Proc. 9. Intern. Conf. on Automatic Fire Detection AUBE'89; Duisburg Univ, 1989 ; p. $245-264$

[15] Okayama, Y:; A Primitive Study on Fire Detection Method Controlled by an Artificial Neural Net, Fire Safety Journal 14 (1991); to be published

* see also in Proc. 9. Intern. Conf. on Automatic Fire Detection AUBE'89 Duisburg University 1989 